\title{
Heat Conduction in Fine Scale Mixtures With Interfacial Contact Resistance
}

\author{
Robert Lipton \\ Department of Mathematical Sciences \\ Worcester Polytechnic Institute \\ 100 Institute Road \\ Worcester, MA 01609
}

\begin{abstract}
Heat conduction in a fine scale mixture of two conductors is examined in the presence of a contact resistance between phases. The problem is studied rigorously in the context of periodic homogenization. Unlike the case of perfect heat transmission between phases, the temperature fields studied here may converge weakly in $L^{2}$ to the homogenized temperature. The temperature gradients converge weakly as Radon measures. The strict ellipticity of the homogenized transport equation depends upon the geometry of the interface. The effective conductivity associated with the overall heat dissipation rate inside a composite cube is considered. It is shown that this property exhibits a size effect under rescaling.
\end{abstract}

Key Words. composite medium, contact resistance, homogenization. AMS (MOS) Subject Classification. 35B27, 35J20, 78A30, 73B27

\section{Introduction}

In this article we investigate the problem of heat transport for finely mixed two phase heat conductors with imperfect heat transmission between phases. We study the effect of a thermal resistance at the two phase interface. This resistance results in a discontinuity in the temperature across the interface. Interfacial resistance can appear as a Kapitza resistance due to the difference in sound velocity and bulk density between the two phases, see [12]. It may also arise due to imperfect bonding between phases, see [6]. To fix ideas, we consider periodic mixtures of two isotropic heat conductors. The geometry within the period cell can be arbitrarily specified. The approach given here is rigorous and is similar in spirit to the theory of periodic homogenization for two phase conductors with perfect heat transmission between phases as presented in Sanchez Palencia [18] and Bensoussan, Lions, and Papanicolaou [4]. 
The composite heat conductor occupies the region $\Omega \subset \mathbb{R}^{3}$. The conductivity of each isotropic phase is specified by $\sigma_{1}$ and $\sigma_{2}$. The period cell is the unit cube $(-1,+1)^{3}$ denoted by $Q$. We introduce the $Q$-periodic characteristic function of phase 1 given by $\chi_{1}(x)$, i.e. $\chi_{1}(x)=1$ if $x$ in phase one, $\chi_{1}(x)=0$ otherwise. The piecewise constant conductivity is defined through

$$
\sigma(x)=\sigma_{1} \chi_{1}(x)+\sigma_{2}\left(1-\chi_{1}(x)\right) .
$$

Rescaling the unit cell $Q$ by the factor $1 / k(k=1,2,3, \ldots)$, we obtain a family of $1 / k$ periodic two phase composite conductors. The regions occupied by materials one and two are denoted by $\Omega_{1}^{k}$ and $\Omega_{2}^{k}$ and the associated conductivity is given by $\sigma^{k}(x)=\sigma(k x)$. The boundary between phases is written as $\Gamma^{k}$ and the phase regions are distributed according to

$$
\Omega=\Omega_{1}^{k} \cup \Omega_{2}^{k} \cup \Gamma^{k} .
$$

In what follows we assume that the interface $\Gamma^{k}$ is Lipschitz. The temperature field for a $1 / k$ periodic composite is denoted by $u^{k}$ and we suppose that $u^{k}=0$ on the boundary of the composite domain $\Omega$. Across the two phase boundary the temperature jumps according to

$$
-\beta\left[u^{k}\right]=\sigma_{1} \nabla u_{1}^{k} \cdot n .
$$

Here $\left[u^{k}\right] \equiv u_{1}^{k}-u_{2}^{k}$ and the subscripts indicate the side of the two-phase boundary where the traces are evaluated. The vector $n$ denotes the unit normal vector pointing into phase 1.

The normal component of the heat-flux is assumed continuous across phase interfaces, i.e.,

$$
\sigma_{1} \nabla u_{1}^{k} \cdot n=\sigma_{2} \nabla u_{2}^{k} \cdot n
$$

For a prescribed heat source $f$ in $L^{2}(\Omega)$ the temperature satisfies:

$$
\begin{array}{ll}
\sigma^{1} \Delta u^{k}=f & \text { in } \Omega_{1}^{k}, \\
\sigma^{2} \Delta u^{k}=f & \text { in } \Omega_{2}^{k} .
\end{array}
$$

Condition (1.3) represents the effect of a thermal resistance at the two phase interface. The parameter $\beta$ denotes the interfacial barrier conductance. The case of perfect contact between phases is recovered in the $\beta=\infty$ limit.

We introduce the function space $U^{k}$ defined by

$$
U^{k}=\left\{\psi \mid \psi=\left(\psi_{1}, \psi_{2}\right) \text {, where } \psi_{1} \text { in } H^{1}\left(\Omega_{1}^{k}\right), \psi_{2} \text { in } H^{1}\left(\Omega_{2}^{k}\right) \text { and } \psi=0 \text { on } \partial \Omega\right\} .
$$

The temperature $u^{k}$ is in $U^{k}$ and the weak formulation of the imperfect heat conduction problem (1.3) - (1.6) is given by

$$
\int_{\Omega_{1}^{k} \cup \Omega_{2}^{k}} \sigma^{k}(x) \nabla u^{k} \cdot \nabla \psi d x+\beta \int_{\Gamma^{k}}\left[u^{k}\right][\psi] d s=\int_{\Omega} \psi f d x
$$


for all $\psi$ in $U^{k}$. Existence of solution for (1.8) follows from direct transposition of arguments given in Lene and Leguillon [14] to the context of heat conductivity and an application of the Lax-Milgram Lemma.

In this paper we provide a homogenization theorem (Theorem 2.1) for general periodic geometries describing the behavior of the temperatures $u^{k}$ in the fine scale limit. Unlike the perfect transmission case, a subsequence of $u^{k}$ converges weakly in $L^{2}(\Omega)$ to the homogenized temperature and the associated temperature gradients converge weakly in the sense of Radon measures, see Theorem 2.1. The strict ellipticity of the homogenized transport equation is seen to depend upon the geometry of the two-phase interface, see Section 7 .

The convergence of the temperatures $u^{k}$ improves if one or both phases are connected. To see this, we denote the subsets of the unit period cell occupied by materials one and two by $Q_{1}^{1}$ and $Q_{2}^{1}$ respectively. We cover $\mathbb{R}^{3}$ with periodic translates of $Q_{1}^{1}$ and denote the union by $Q_{1}^{*}$. To fix ideas we make the following hypotheses on the region occupied by material one:

(H1) $Q_{1}^{1}$ is an open connected subset of $\mathbb{R}^{3}$ and has Lipschitz boundary.

(H2) $Q_{1}^{*}$ is connected and has Lipschitz boundary.

With these hypotheses we show there exists a "homogenized" temperature $u^{0}$ in $H_{0}^{1}(\Omega)$ such that

$$
\left\|u^{k}-u^{0}\right\|_{L^{2}(\Omega)} \rightarrow 0
$$

Here $u^{0}$ is the unique solution to the homogenized transport equation, see Theorem 2.3.

The homogenization theorems developed here agree with the behavior suggested by recent bounds on the effective conductivity of composites with imperfect interface given by Lipton and Vernescu [16]. In that work the composite consisted of the unit cube filled with an isotropic suspension of particles of conductivity $\sigma_{2}$ in a matrix of $\sigma_{1}$ such that $\sigma_{2}>\sigma_{1}$. For this case the lower bound given by equation (II.2.10) of [16] is seen to admit the expansion.

$$
\tilde{\sigma}^{e}+O(1 / S)
$$

Here $S$ is the interfacial surface area. The quantity $\tilde{\sigma}^{e}$ is the effective heat conductivity of a suspension with the same geometry but with particles filled by a perfectly insulating material. In Section 3 we consider a unit cube filled with a $1 / k$ periodic arrangement of particles and show that the associated effective property $\sigma_{k}^{e}$ has the same asymptotic behavior as the lower bound (1.9) in the $k=\infty$ limit, see Remarks 3.5 and 3.6.

In Section 3, Theorem 3.3 we demonstrate the existence of a size effect. That is, we show that the effective conductivity of $1 / k$ periodic composite with barrier conductance $\beta$ is identical to that of unit periodic composite with barrier conductance $\beta / k$. This result is in sharp contrast to the invariance under rescaling enjoyed by the effective properties 
of periodic composites when there is perfect heat transmission between phases. In fact it is shown in Theorem 3.4 that the effective conductivity is decreasing with the scale of periodicity. Thus, given a 1 periodic local conductivity $\sigma(x)$ we see that pulverizing the geometry to obtain a finer mixture $\sigma(k x)$ only serves to decrease the effective conductivity. Physically, this is accounted for by the increased surface area of the interface.

Earlier work of Lene and Leguillon [15] treated the case of isolated elastic inclusions in a connected elastic matrix with slip at the two-phase interface. Unlike this presentation, they allow the interfacial properties to change with the period of the composite. In the context of heat conductivity, their problem corresponds to a barrier conductance that increases as the scale of the period decreases. Their homogenization proof makes elegant use of a uniformly bounded family of extension operators. These operators continuously extend deformation fields from the matrix into the inclusions. Due to the construction of these operators, their approach is limited to matrix inclusion composites. In contrast, the approach developed here makes no use of extension operators and applies to arbitrary periodic geometries. For the special case when hypothesis (H1) and (H2) hold, we employ the compactness Lemma of Allaire, Murat, and Nandakumar [1]. The lemma is used to show that the sequence $\left\{u^{k}\right\}$ converges strongly in $L^{2}$ to the solution of the homogenized problem.

We emphasize that the geometric hypotheses (H1) and (H2) cover a wide range of cases, since they allow the second phase to have connected as well as disconnected components.

The methods used in this analysis can be applied to the rigorous homogenization of two-phase elastic structures with interfacial slip. Indeed, if the coefficient of interfacial slip is held fixed for all scales of periodicity, then one recovers results analagous to those given in this paper. If the coefficient of slip is allowed to increase inversely proportional to the periodicity of the composite, then it is possible to extend the results of Lene and Leguillon [15] to arbitrary two-phase, periodic, elastic composite geometries.

The paper is organized as follows: In the following Section we state the homogenization theorems. In Section 3 we introduce correctors and define the effective heat conductivity tensor. We establish the homogenization theorem for the special case when the composite domain is the unit cube and the average heat intensity is prescribed, see Theorem 3.4.

We apply these results in our treatment of periodic homogenization for arbitrary composite domains. Section 4 is devoted to obtaining uniform bounds on the sequences $\left\{u^{k}\right\}$, $\left\{\nabla u^{k}\right\}$, and $\left\{\left[u^{k}\right]\right\}$. Apriori estimates are accomplished through a Poincaré like inequality for the spaces $U^{k}$, see Theorem 4.1. The weak limit $M^{\circ}$ of the sequence of heat flux vectors $\left\{\sigma^{k} \nabla u^{k}\right\}$ is shown to satisfy a macroscopic balance equation, see (4.17). In Section 5 the two-scale limit of the temperatures $\left\{u^{k}\right\}$, denoted by $u^{\circ}(x, y)$, is shown to depend only upon the macroscopic variable " $x$ ". In Section 6 we apply the two-scale convergence theorem of Nguetseng [17] to identify the weak limit of the heat flux vectors. We conclude the proof of the homogenization theorem for general periodic geometries in Section 7 . There we discuss the link between strict elipticity of the homogenized problem and the geometry of the 
two-phase interface. It is found that these questions are intimately related to homogenization theorems for perforated domains. We appeal to a theorem of Briane [7] for perforated domains to show that the homogenized problem is strictly elliptic for composite geometries satisfying (H1) and (H2).

In Section 8 we suppose material one is connected according to (H1) and (H2). We apply the compactness Lemma of Allaire, Murat and Nandakumar [1] to establish the existence of a strong $L^{2}$ convergent sequence of temperature fields. From this, one easily obtains Theorem 2.3 using the methods of Sections 6 and 7 .

\section{Periodic Homogenization Theorems}

We present two homogenization theorems. To fix ideas we begin with a homogenization theorem for general periodic geometries. The two-phase interface is given by $\gamma^{1}$ and the unit cell is partitioned according to:

$$
Q=Q_{1}^{1} \cup Q_{2}^{1} \cup \gamma^{1}
$$

where the Lebesque measure of $\gamma^{1}$ is zero. It is shown in Section 4 (see Remark 4.4) that the solutions $u^{k}$ of the heat conductivity problems (1.6) are elements of the space $S B V(\Omega)$ introduced by Ambrosio and DeGeorgi in [2]. The distributional gradient of $u^{k}$ is a Radon measure denoted by $D u^{k}$. It has the representation

$$
D u^{k}=\nabla u^{k} d x-\left[u^{k}\right] n d H_{2}\left\lfloor\Gamma^{k}\right.
$$

where $\nabla u^{k}$ lies in $L^{2}(\Omega)^{3}$ and is the absolutely continuous part of $D u^{k}$ with respect to 3

dimensional Lebesque measure and $H_{2}$ is 2 dimensional Hausdorff measure. Since $\Gamma^{k}$ is Lipschitz, $d H_{2}\left\lfloor\Gamma^{k}\right.$ corresponds to surface measure.

For a periodic arrangement of two conductors we introduce the symmetric tensors $\mathbf{g}^{1}$ and $\mathbf{g}^{2}$ defined by

$$
\mathbf{g}^{1} \xi \cdot \xi=\inf _{\psi \in V^{1}} \int_{Q_{1}^{1}}|\nabla \psi+\xi|^{2} d x
$$

and

$$
\mathbf{g}^{2} \xi \cdot \xi=\inf _{\psi \in V^{1}} \int_{Q_{2}^{1}}|\nabla \psi+\xi|^{2} d x
$$

for any $\xi$ in $\mathbb{R}^{3}$. The space $V^{1}$ is defined as

$$
V^{1}=\left\{\psi-Q \text { periodic } \mid \psi=\left(\psi_{1}, \psi_{2}\right), \psi_{1} \text { in } H^{1}\left(Q_{1}^{1}\right), \psi_{2} \text { in } H^{1}\left(Q_{2}^{1}\right)\right\}
$$

and the geometric tensors $\mathbf{g}^{j}, j=1,2$ represent the effective conductivity of a composite with material of unit conductivity in $Q_{j}^{1}$ and a perfect insulator in the complement. The 
minimizers $\psi_{j}^{\infty}, j=1,2$ of $(2.3)$ and $(2.4)$ are unique up to a constant and satisfy the following equilibrium equation

$$
\int_{Q_{j}^{1}}\left(\nabla \psi_{j}^{\infty}+\xi\right) \cdot \nabla \delta d x=0
$$

for all $\delta$ in $V^{1}$, and

$$
\mathbf{g}^{1} \xi \cdot \xi=\int_{Q_{1}^{1}}\left|\nabla \psi_{1}^{\infty}+\xi\right|^{2} d x, \quad \mathbf{g}^{2} \xi \cdot \xi=\int_{Q_{2}^{1}}\left|\nabla \psi_{2}^{\infty}+\xi\right|^{2} d x .
$$

We mention that the inverses of the tensors $\mathbf{g}^{1}$ and $\mathbf{g}^{2}$ are the formation factor tensors associated with phases one and two respectively. Such tensors are routinely used in the study of porous media, cf.,Dullien [8].

Introducing the positive semidefinite tensor

$$
\mathbf{c}=\sigma_{1} \mathbf{g}^{1}+\sigma_{2} \mathbf{g}^{2}
$$

we have the following:

Theorem 2.1. Homogenization of Periodic Composites with Imperfect Interface.

For any $f$ in $L^{2}(\Omega)$ there exists a temperature field $u^{\circ}$ and a subsequence $u^{k}$ for which:

$$
u^{k} \rightarrow u^{0} \text { weakly in } L^{2}(\Omega)
$$

and $u^{\circ}$ is a local solution of

$$
-\operatorname{div}\left(\boldsymbol{c} \nabla u^{0}\right)=f
$$

Moreover, if $\boldsymbol{c}$ is positive definite, then $u^{\circ}$ lies in $H^{1}(\Omega)$ and the distributions $D u^{k}$ converge weakly to $\nabla u^{\circ}$ in the sense of Radon measures, i.e.,

$$
D u^{k} \rightarrow \nabla u^{0} d x
$$

Remark 2.2. The convergence of the distributions $D u^{k}$ implied by (2.11) reads

$$
\int_{\Omega} \psi \cdot d\left[D u^{k}\right] \rightarrow \int_{\Omega} \psi \cdot \nabla u^{0} d x
$$

for all $\psi$ in $C_{c}^{1}(\Omega)$.

Next we assume that material one is distributed according to the hypotheses (H1) and (H2). An application of Proposition 5.1 of Briane [7] shows that the homogenized tensor c is positive definite, (see Section 7). For this case we have: 
Theorem 2.3. Given any $f$ in $L^{2}(\Omega)$ there exists $u^{0}$ in $H_{0}^{1}(\Omega)$ for which the solutions $u^{k}$ of problems (1.8) converge in the following sense:

$$
u^{k} \rightarrow u^{0} \text { strongly in } L^{2}(\Omega)
$$

and the distributions $D u^{k}$ converge weakly to $\nabla u^{\circ}$ in the sense of Radon measures. Moreover, $u^{\circ}$ is the unique solution of

$$
-\operatorname{div}\left(\boldsymbol{c} \nabla u^{0}\right)=f
$$

\section{Correctors, Effective Conductivity, and Homogenization of Periodic Composites in the Unit Cube}

In this Section we introduce the effective conductivity tensor for a $1 / k$ periodic composite occupying the unit cube $Q$. We introduce the associated temperature fields (referred to as correctors) and provide a homogenization theorem describing heat transport in the fine phase limit. This result is essential for establishing Theorems 2.1 and 2.3.

To be precise, we suppose that the cube $Q$ is filled with a $1 / k$ periodic composite with local conductivity $\sigma^{k}(x)=\sigma(k x)$. The regions occupied by materials one and two are denoted by $Q_{1}^{k}$ and $Q_{2}^{k}$ respectively. The two phase interface denoted by $\gamma^{k}$ has Lebesque measure zero and $Q=Q_{1}^{k} \cup Q_{2}^{k} \cup \gamma^{k}$ (for $k=1,2,3 \ldots$ ). We introduce the associated function spaces $V^{k}$ given by

$$
V^{k}=\left\{\psi-Q \text { periodic } \mid \psi=\left(\psi_{1} \psi_{2}\right), \psi_{1} \text { in } H^{1}\left(Q_{1}^{k}\right), \psi_{2} \text { in } H^{1}\left(Q_{2}^{k}\right)\right\}
$$

(for $k=1,2, \ldots$ ).

We consider first a composite with periodicity one and the local conductivity given by $\sigma(x)$ and interfacial barrier conductance $\beta / \ell,(\ell=1,2,3 \ldots)$. For fixed $\ell$ the temperature in the composite can be written as the sum of a periodic fluctuation $\psi^{\ell}$ and a linear function $\xi \cdot x$. The constant vector $\xi$ in $R^{3}$ is prescribed and represents the average heat intensity as measured by an observer outside the composite, i.e.

$$
\int_{\partial Q}\left(\psi^{\ell}+\xi \cdot x\right) n d s=\xi .
$$

See Benveniste [5]. The periodic fluctuation $\psi^{\ell}$ lies in the function space $V^{1}$ and is a solution of the thermal equilibrium, equations:

$$
\begin{aligned}
& \Delta \psi^{\ell}=0 \text { in } \quad Q_{1}^{1} \cup Q_{2}^{1} \\
& n \cdot \sigma_{1}\left(\nabla \psi_{1}^{\ell}+\xi\right)=n \cdot \sigma_{2}\left(\nabla \psi^{\ell}+\xi\right) \text { on } \gamma^{1}, \\
& n \cdot \sigma_{1}\left(\nabla \psi_{1}^{\ell}+\xi\right)=-\beta / \ell\left[\psi^{\ell}\right] \text { on } \gamma^{1}
\end{aligned}
$$


Here $n$ is the unit normal pointing into phase 1.

The solutions $\psi^{\ell}$ of (3.3) - (3.5) exist and are unique up to a constant; this is an application of the Lax-Milgram lemma. For future reference, the variational form of (3.3) $(3.5)$ is given by

$$
\int_{Q_{1}^{1} \cup Q_{2}^{1}} \sigma(x)\left(\nabla \psi^{\ell}+\xi\right) \cdot \nabla \delta d x+\beta / \ell \int_{\gamma^{1}}\left[\psi^{\ell}\right][\delta] d s=0
$$

for all $\delta$ in $V^{1}$.

The effective conductivity $\tilde{\sigma}^{\ell}$ of the two phase composite is defined by

$$
\tilde{\sigma}^{\ell} \xi=\int_{Q} \sigma(x)\left(\nabla \psi^{\ell}+\xi\right) d x
$$

see Benveniste [5]. Introducing the Lagrangian $L_{\ell}(\psi)$ defined by

$$
L_{\ell}(\psi)=\int_{Q_{1}^{1} \cup Q_{2}^{1}} \sigma(x)|\nabla \psi+\xi|^{2}+\beta / \ell \int_{\gamma^{1}}([\psi])^{2} d s,
$$

one has that the effective conductivity $\tilde{\sigma}^{\ell}$ admits the equivalent variational representation

$$
\tilde{\sigma}^{\ell} \xi \cdot \xi=\inf _{\psi \in V^{1}} L_{\ell}(\psi)
$$

and $\tilde{\sigma}^{\ell} \xi \cdot \xi=L_{\ell}\left(\psi^{\ell}\right)$.

Remark 3.1. It is easily seen from (3.8) that the energies $\tilde{\sigma}^{\ell} \xi \cdot \xi$ are monotone decreasing in $\ell$ and

$$
\tilde{\sigma}^{\ell} \xi \cdot \xi \geq \sigma_{1} \mathbf{g}^{1} \xi \cdot \xi+\sigma_{2} \mathbf{g}^{2} \xi \cdot \xi
$$

for the tensors $\mathbf{g}^{1}, \mathbf{g}^{2}$ given by (2.7) and (2.8). Moreover, since $\tilde{\sigma}^{\ell} \xi \cdot \xi$ is the infimum of the family of convex functions $L_{\ell}(\psi)$, it follows that $\tilde{\sigma}^{\ell} \xi \cdot \xi$ is upper semicontinuous in $\ell$ and we conclude:

$$
\lim _{\ell \rightarrow \infty} \tilde{\sigma}^{\ell} \xi \cdot \xi=\sigma_{1} \mathbf{g}^{1} \xi \cdot \xi+\sigma_{2} \mathbf{g}^{2} \xi \cdot \xi .
$$

We introduce the minimizers $\psi_{1}^{\infty}, \psi_{2}^{\infty}$ of (2.3) and (2.4) respectively and state the following

\section{Lemma 3.2.}

$$
\nabla \psi_{j}^{\ell}+\xi \rightarrow \nabla \psi_{j}^{\infty}+\xi, \quad j=1,2
$$

strongly in $L^{2}\left(Q_{j}^{1}\right)^{3}$.

Here $\psi_{j}^{\ell}$ represents the component of $\psi^{\ell}$ in the phase region $Q_{j}^{1}, j=1,2$.

Proof: We start by showing

$$
\lim _{\ell \rightarrow \infty} \beta / \ell \int_{\gamma^{1}}\left(\left[\psi^{\ell}\right]\right)^{2} d s=0 .
$$


Noting as before that $\tilde{\sigma}^{\ell} \xi \cdot \xi=L_{\ell}\left(\psi^{\ell}\right)$ we write

$$
\beta / \ell \int_{\gamma^{1}}\left(\left[\psi^{\ell}\right]\right)^{2} d s=\tilde{\sigma}^{\ell} \xi \cdot \xi \cdot \xi-\int_{Q_{1}^{1} \cup Q_{2}^{1}} \sigma(x)\left|\nabla \psi^{\ell}+\xi\right|^{2} .
$$

From (3.11) and (3.14) it is evident that

$$
\lim _{\ell \rightarrow \infty} \beta / \ell \int_{\gamma^{1}}\left(\left[\psi^{\ell}\right]\right)^{2} d s=\sigma_{1} \mathbf{g}^{1} \xi \cdot \xi+\sigma_{2} \mathbf{g}^{2} \xi \cdot \xi-\lim _{\ell \rightarrow \infty} \int_{Q_{1}^{1} \cup Q_{2}^{1}} \sigma(x)\left|\nabla \psi^{\ell}+\xi\right|^{2} d x .
$$

Since (2.3) and (2.4) imply

$$
\sigma_{1} \mathbf{g}^{1} \xi \cdot \xi+\sigma_{2} \mathbf{g}^{2} \xi \cdot \xi=\inf _{\psi \in V^{1}}\left(\sigma_{1} \int_{Q_{1}^{1}}|\nabla \psi+\xi|^{2} d x+\sigma_{2} \int_{Q_{2}^{1}}|\nabla \psi+\xi|^{2} d x\right)
$$

we see that for all $\ell$ that

$$
\sigma_{1} \mathbf{g}^{1} \xi \cdot \xi+\sigma_{2} \mathbf{g}^{2} \xi \cdot \xi \leq \int_{Q_{1}^{1} \cup Q_{2}^{1}} \sigma(x)\left|\nabla \psi^{\ell}+\xi\right|^{2} d x .
$$

It is apparent from (3.15) and (3.17) that

$$
\lim _{\ell \rightarrow \infty} \beta / \ell \int_{\gamma^{1}}\left(\left[\psi^{\ell}\right]\right)^{2} d s \leq 0
$$

and the desired results follows immediately from the positivity of

$$
\beta / \ell \int_{\gamma^{1}}\left(\left[\psi^{\ell}\right]\right)^{2} d s .
$$

It is easy to see from (3.13) and (3.15) that

$$
\lim _{\ell \rightarrow \infty} \int_{Q_{1}^{1} \cup Q_{2}^{1}} \sigma(x)\left|\nabla \psi^{\ell}+\xi\right|^{2} d x=\sigma_{1} \mathbf{g}^{1} \xi \cdot \xi+\sigma_{2} \mathbf{g}^{2} \xi \cdot \xi .
$$

Noting that $\sigma(x) \geq \min \left(\sigma_{1}, \sigma_{2}\right)>0$, it follows from $(3,20)$ togeather with $(2.3),(2,4)$, and (2.7) that

$$
\lim _{\ell \rightarrow \infty} \int_{Q_{j}^{1}}\left|\nabla \psi_{j}^{\ell}+\xi\right|^{2} d x=\int_{Q_{j}^{1}}\left|\nabla \psi_{j}^{\infty}+\xi\right|^{2} d x .
$$

To finish the proof we show $\nabla \psi_{j}^{\ell}+\xi \rightarrow \nabla \psi_{j}^{\infty}+\xi$ weakly in $L^{2}\left(\left[Q_{j}^{1}\right]\right)^{3}$. This is done in two steps: First we show that subsequence $\psi_{j}^{\ell_{K}}$ (with each term adjusted by a constant if necessary) converges weakly in $H^{1}\left(Q_{1}^{1}\right)$ to a limit $\tilde{\psi}_{j}^{\infty}$. From this it follows that $\nabla \psi_{j}^{\ell_{K}}-$ $\nabla \tilde{\psi}_{j}^{\infty}$ weakly in $L^{2}\left(Q_{j}^{1}\right)^{3}$. Second, we pass to the limit in (3.6) noting that the solutions $\psi_{j}^{\infty}, j=1,2$ of (2.6) are unique up to a constant. From this, we conclude that the whole sequence $\nabla \psi_{j}^{\ell}+\xi$ converges to $\nabla \psi_{j}^{\infty}+\xi$ weakly in $L^{2}\left(\left[Q_{j}^{1}\right]\right)^{3}$. 
To show the first part we observe that (3.21) implies that the sequence $\left\{\nabla \psi_{j}^{\ell}\right\}$ is uniformly bounded in $L^{2}\left(Q_{j}^{1}\right)$. We focus on the phase region $Q_{1}^{1}$. Subtracting an appropriate constant (if necessary) from the component $\psi_{1}^{\ell}$ we apply Poincaré's inequality to show that $\left\{\psi_{1}^{\ell}\right\}_{\ell}$ is uniformly bounded in $L^{2}\left(Q_{1}^{1}\right)$, hence $\left\{\psi_{1}^{\ell}\right\}_{\ell}$ is uniformly bounded in $H^{1}\left(Q_{1}^{1}\right)$. From the Rellich Kondrachov compactness theorem we conclude existence of a subsequence $\nabla \psi_{1}^{\ell_{K}}$ converging weakly to $\nabla \tilde{\psi}_{1}^{\infty}$ with $\tilde{\psi}_{1}^{\infty}$ in $H^{1}\left(Q_{1}^{1}\right)$. We pass to the limit in the variational formulation (3.6), applying (3.13) to find that $\tilde{\psi}_{1}^{\infty}$ is the solution of (2.6). From uniqueness it follows that $\nabla \tilde{\psi}_{1}^{\infty}=\nabla \psi_{1}^{\infty}$ and the whole sequence $\nabla \psi_{1}^{\ell}$ converses weakly to $\nabla \psi_{1}^{\infty}$. Identical arguments can be made for the sequence $\left\{\psi_{2}^{\ell}\right\}_{\ell}$ and the Lemma is established.

We extend our analysis to $1 / k$-periodic composites with scale independent interfacial boundary conductance given by $\beta$. For prescribed heat intensity $\xi$ and for a $1 / k$-periodic arrangement of conductors specified by $\sigma^{k}(x)=\sigma(k x)$, the associated temperature field is written $\phi^{k}=w^{k}+\xi \cdot x$. Here $w^{k}$ is an element of $V^{k}$ and $\phi^{k}$ is the solution of

$$
\begin{aligned}
& \nabla \cdot \sigma^{k}\left(\nabla \phi^{k}\right)=0 \quad \text { in } Q_{j}^{k} \quad j=1,2 \\
& n \cdot \sigma_{1} \nabla \phi_{1}^{k}=n \cdot \sigma_{2} \nabla \phi_{2}^{k} \quad \text { on } \gamma^{k}
\end{aligned}
$$

and

$$
-\beta\left(\phi_{1}^{k}-\phi_{2}^{k}\right)=n \cdot \sigma_{1} \nabla \phi_{1}^{k} \quad \text { on } \gamma^{k} .
$$

It is possible to write the solution $\phi^{k}$ in terms of the solution of (3.6) for a unit periodic geometry with interfacial barrier conductance $\beta / k$. Indeed extending the solution $\psi^{k}$ of (3.6) by periodicity to $\mathbb{R}^{3}$ we find that

$$
\phi^{k}=k^{-1}\left(\psi^{k}(k x)+\xi \cdot(k x)\right) .
$$

This follows immediately by rescaling in (3.3) - (3.5) for $\ell=k$. Writing $y=k x$ we have $\nabla \phi^{k}=\left(\nabla_{y} \psi_{j}^{k}(k x)+\xi\right)$ in $Q_{j}^{1}$. Here $\nabla_{y}$ indicates a gradient with respect to $y$. We introduce the energy associated with the corrector $\nabla \phi^{k}$ given by

$$
E\left(\phi^{k}\right)=\int_{Q_{1}^{k} \cup Q_{2}^{k}} \sigma^{k}(x)\left|\nabla \phi^{k}\right|^{2}+\beta \int_{\gamma^{k}}\left(\left[\phi^{k}\right]\right)^{2} d s .
$$

This energy is precisely that defining the effective conductivity of a $1 / k$-periodic composite with interfacial barrier conductance $\beta$. We denote the effective conductivity for this composite by $\sigma_{k}^{e} \xi \cdot \xi$ and from its definition we have

$$
\sigma_{k}^{e} \xi \cdot \xi=E\left(\phi^{k}\right)
$$

We now state the following:

Theorem 3.3. Size Effect for Periodic Composites. 
The effective conductivity of a composite with local conductivity $\sigma(x)$ and interfacial barrier conductance $\beta / k$ is identical to that of a $1 / k$ periodic composite with local conductivity $\sigma^{k}(x)=\sigma(k x)$ and interfacial barrier conductance $\beta$, i.e.

$$
\sigma_{k}^{e}=\tilde{\sigma}^{k}
$$

Proof: The theorem follows from (3.25) and rescaling in equation (3.26).

From Theorem 3.3 and Lemma 3.2 we have the following homogenization theorem for periodic composites in the unit cell.

Theorem 3.4. Homogenization of the Cell Problem.

For a prescribed average intensity $\xi$ :

$$
\sigma_{k}^{e} \xi \cdot \xi \geq \sigma_{k+1}^{c} \xi \cdot \xi
$$

and

$$
\lim _{k \rightarrow \infty} \sigma_{k}^{e} \xi \cdot \xi=\left(\sigma_{1} \mathbf{g}^{1}+\sigma_{2} \mathbf{g}^{2}\right) \xi \cdot \xi .
$$

Moreover the associated periodic intensities

$$
\begin{aligned}
\nabla \phi^{k} & = \begin{cases}\nabla_{y} \psi_{1}^{k}(k x)+\xi & \text { in } Q_{1}^{k} \\
\nabla_{y} \psi_{2}^{k}(k x)+\xi & \text { in } Q_{2}^{k}\end{cases} \\
\nabla \phi^{\infty} & = \begin{cases}\nabla \psi_{1}^{\infty}+\xi & \text { in } Q_{1}^{1} \\
\nabla \psi_{2}^{\infty}+\xi & \text { in } Q_{2}^{1}\end{cases}
\end{aligned}
$$

are elements of $L^{2}(Q)^{3}$ and

$$
\nabla \phi^{k}-\int_{Q} \nabla \phi^{\infty} d y, \text { weakly in } L^{2}(Q)^{3},
$$

and

$$
\sigma^{k} \nabla \phi^{k}-\left(\sigma_{1} \mathbf{g}^{1}+\sigma_{2} \mathbf{g}^{2}\right) \xi, \text { weakly in } L^{2}(Q)^{3}
$$

Proof: The monotonicity expressed by (3.29) follows from Remark 3.1 and Theorem 3.3.

The convergence of energies given by (3.30) is a consequence of Theorem 3.3 and (3.11). Since the measure of the interface $\gamma^{k}$ is zero it follows that $\nabla \phi^{k}$ and $\nabla \phi^{\infty}$ are elements of $L^{2}(Q)^{3}$. We introduce the sequence $\left\{\nabla \phi^{\infty, k}(x)\right\}$ defined by $\nabla \phi^{\infty, k}(x)=\nabla \phi^{\infty}(k x)$ and

$$
\left\|\nabla \phi^{\infty, k}(x)-\nabla \phi^{k}(x)\right\|_{L^{2}(Q)^{3}}^{2}=\sum_{j=1}^{2} \int_{Q_{j}^{1}}\left|\nabla_{y} \psi_{j}^{\infty}(y)-\nabla_{y} \psi_{j}^{k}(y)\right|^{2} d y .
$$


It is evident from (3.35) and Lemma (3.2) that the difference $\nabla \phi^{\infty, k}-\nabla \phi^{k}$ converges strongly in $L^{2}(Q)^{3}$ to zero. On the other hand

$$
\nabla \phi^{\infty, k}-\int_{Q} \nabla \phi^{\infty} d y
$$

weakly in $L^{2}(Q)^{3}$. For any vector $\tau(x)$ in $L^{2}(Q)^{3}$ we write

$$
\int_{Q} \nabla \phi^{k} \cdot \tau d x=\int_{Q}\left(\nabla \phi^{k}-\nabla \phi^{\infty, k}\right) \cdot \tau d x+\int_{Q} \nabla \phi^{\infty, k} \cdot \tau d x .
$$

Equation (3.33) follows immediately by passing to the limit in (3.37) using (3.36) and the strong $L^{2}(Q)^{3}$ convergence of $\nabla \phi^{\infty, k}-\nabla \phi^{k}$. The weak convergence result (3.34) follows from an identical proof noting that

$$
\sigma^{k} \nabla \phi^{\infty, k}-\int_{Q} \sigma(x) \nabla \phi^{\infty} d x=\left(\sigma_{1} \mathbf{g}^{1}+\sigma_{2} \mathbf{g}^{2}\right) \xi
$$

weakly in $L^{2}(Q)^{3}$.

Remark 3.5. We consider suspensions of isolated particles of material two in a matrix of material one. Calculation shows that the minimizer of (2.8) is of the form $\psi_{2}^{\infty}=-\xi \cdot x$ and so $\mathbf{g}^{2}=0$. Hence Theorem 3.4 yields:

$$
\lim _{k \rightarrow \infty} \sigma_{k}^{e}=\sigma_{1} \mathbf{g}^{1}
$$

Remark 3.6. From well known homogeneity properties (c.f. Golden and Papanicolaou [13]), one has $\tilde{\sigma}^{e}=\sigma_{1} \mathbf{g}^{1}$ where $\tilde{\sigma}^{e}$ is the effective conductivity associated with the same geometry but with a matrix of conductivity $\sigma^{1}$. Thus the convergence given by (3.30) shows that the effective tensors $\sigma_{k}^{e}$ exhibit the same asymptotic behavior as the lower bound given by (1.9).

We conclude this Section with the following useful estimate:

Theorem 3.7. There exists a constant $C$ independent of $k$ such that:

$$
\left\|k^{-1} \psi^{k}(y)\right\|_{L^{2}(Q)} \leq k^{-1 / 2} C
$$

The proof is obtained using:

Proposition 3.8. The following Poincaré-like inequality holds

$$
\int_{Q_{1}^{1} \cup Q_{2}^{1}} \psi^{2} d x \leq M\left\{\int_{Q_{1}^{1} \cup Q_{2}^{1}}|\nabla \psi|^{2} d x+\int_{\gamma^{1}}([\psi])^{2} d s\right\}
$$


for every $\psi$ in $V^{1}$ such that $\int_{Q_{1}^{1} \cup Q_{2}^{1}} \psi d x=0$ and an appropriate constant $M$.

Proof: The proposition is easily established by contradiction.

From (3.13) and (3.21) it follows that

$$
\sup _{k}\left\{k^{-1} \int_{\gamma^{1}}\left(\left[\psi^{k}\right]\right)^{2} d s\right\}<\infty, \quad \sup _{k} \int_{Q_{1}^{1} \cup Q_{2}^{1}}\left|\nabla \psi^{k}\right|^{2} d x<\infty
$$

and Theorem 3.7 follows directly from substitution of $k^{-1} \psi^{k}$ into (3.40).

\section{Estimates and the Homogenized Equilibrium Law for the Heat Flux}

To establish Theorems 2.1 and 2.3 we find uniform estimates for the sequence $\left\{u^{k}\right\}_{k}$. We use the following Poincaré-type inequality:

Theorem 4.1. For each $k=1,2, \ldots$ the functions $v$ in $U^{k}$ are elements in $L^{2}(\Omega)$ and

$$
\|v\|_{L^{2}(\Omega)}^{2} \leq C\left\{\int_{\Omega_{1}^{k} \cup \Omega_{2}^{k}}|\nabla v|^{2}+\int_{\Gamma^{K}}([v])^{2} d s\right\}
$$

for all $v$ in $U^{k}$.

Here the constant $C$ is independent of the index $k$ and

$$
C=\max \left\{2(\text { diameter } \Omega)^{2}, \quad 4(\text { diameter } \Omega)\right\}
$$

Proof: Since $v$ lies in $U^{k}$ and meas $\left(\Gamma^{k}\right)=0$, it is evident that $v$ belongs to $L^{2}(\Omega)$. Translating $\Omega$ if necessary, we enclose it in a cube centered at the origin with edge length $2 a=$ diameter $\Omega$. For a fixed index " $k$ " we start by considering functions $v$ in $C^{1}\left(\Omega_{1}^{k}\right) \cup$ $C^{1}\left(\Omega_{1}^{k}\right) \cap U^{k}$. We denote the pair $\left(x_{2}, x_{3}\right)$ by $x^{\prime}$. For fixed $x^{\prime}$ we list all values of the $x_{1}$ coordinate for which the point $x$ lies on the two-phase interface. These are denoted by $\alpha_{N}<\alpha_{N-1}<\cdots \alpha_{2}<\alpha_{1}$. Since the interfaces are assumed Lipschitz, the $x_{1}$-coordinates $\left\{\alpha_{j}\right\}_{j=1}^{N}$ are Lipschitz functions of $x^{\prime}$ and the $j$ th component of the 2-phase interface is given by $x_{1}=\alpha_{j}\left(x^{\prime}\right), j=1,2 \ldots N$. For any point $x$ in $\Omega$ with $x^{\prime}$ fixed we have the identity.

$$
\begin{aligned}
& v\left(x_{1}, x^{\prime}\right)=v\left(x_{1}, x^{\prime}\right)-v\left(\alpha_{1}, x^{\prime}\right)+\sum_{j=1}^{N}\left[v\left(\alpha_{j}, x^{\prime}\right)\right]_{-}^{+} \\
& +\sum_{j=1}^{N-1} v\left(\alpha_{j}, x^{\prime}\right)^{-}-v\left(\alpha_{j+1}, x^{\prime}\right)^{+}+v\left(\alpha_{N}, x^{\prime}\right)^{-}-v\left(\alpha^{*}, x^{\prime}\right) .
\end{aligned}
$$


Here "+" indicates a trace taken from the right of the interface and "-" is that from the left. The point $\left(\alpha^{*}, x^{\prime}\right)$ is chosen to lie on $\delta \Omega$ and $v=0$ there. Each term under the second summation sign in (4.3) is written

$$
v\left(\alpha_{j}, x^{\prime}\right)^{-}-v\left(\alpha_{j+1}, x^{\prime}\right)^{+}=\int_{\alpha_{j+1}}^{\alpha_{j}} \frac{d}{d t} v\left(t, x^{\prime}\right) d t
$$

Extending the function $v$ by zero outside $\Omega$ we have

$$
v\left(x_{1}, x^{\prime}\right)=\int_{-a}^{x_{1}} \frac{d}{d t} v\left(t, x^{\prime}\right) d t+\sum_{j=1}^{N}\left[v\left(\alpha_{j}, x^{\prime}\right)\right]_{-}^{+}
$$

Application of Cauchy's inequality gives

$$
v^{2}\left(x_{1}, x^{\prime}\right) \leq 2\left(2 a \int_{-a}^{a}\left(\partial_{x_{1}} v\left(t, x^{\prime}\right)\right)^{2} d t+\sum_{j=1}^{N}\left(\left[v\left(\alpha_{j}, x^{\prime}\right)\right]_{-}^{+}\right)^{2}\right)
$$

Integrating (4.6) with respect to $x_{1}$ and $x^{\prime}$ over the cube gives

$$
\int_{\Omega} v^{2} d x \leq 2\left(4 a^{2} \int_{\Omega}\left(\partial_{x_{1}} v\right)^{2} d x+4 a \sum_{j=1}^{N} \int_{-a}^{a} \int_{-a}^{a}\left(\left[v\left(\alpha_{j}, x^{\prime}\right)\right]\right)^{2} d x^{\prime}\right) .
$$

On the two-phase interface $x_{1}=\alpha_{j}\left(x^{\prime}\right)$ and we have $i \cdot n d s=d x^{\prime}$, where $i$ is the unit vector along the $x_{1}$ axis. Noting that $|i \cdot n| \leq 1$ we have

$$
\int_{\Omega} v^{2} d x \leq 2\left(4 a^{2} \int_{\Omega}\left(\partial_{x_{1}} v\right)^{2} d x+4 a \int_{\Gamma^{k}}([v])^{2} d s\right)
$$

which implies (4.1). The estimate (4.1) is extended to $U^{k}$ using standard density and trace theorems, for $H^{1}\left(\Omega_{1}^{k}\right)$ and $H^{1}\left(\Omega_{2}^{k}\right)$.

We obtain the necessary estimates given by

Theorem 4.2. The sequence of temperatures $\left\{u^{k}\right\}$ satisfy the estimates

$$
\begin{aligned}
& \sup _{k}\left\|u^{k}\right\|_{L^{2}(\Omega)}<\infty, \\
& \sup _{k}\left\|\nabla u^{k}\right\|_{L^{2}\left(\Omega_{1}^{k} \cup \Omega_{2}^{k}\right)}<\infty, \\
& \sup _{k} \int_{\Gamma^{k}}\left(\left[u^{k}\right]\right)^{2} d s<\infty,
\end{aligned}
$$

and

$$
\sup _{k}\left\|\sigma^{k} \nabla u^{k}\right\|_{L^{2}\left(\Omega_{1}^{k} \cup \Omega_{2}^{k}\right)}<\infty
$$


Proof: For the choice $\psi=u^{k}$ in (1.8) we obtain

$$
\int_{\Omega_{1}^{k} \cup \Omega_{k 2}^{K}} \sigma^{k}\left|\nabla u^{k}\right|^{2} d x+\beta \int_{\Gamma^{k}}\left(\left[u^{k}\right]\right)^{2} d s=\int_{\Omega} u^{k} f d x .
$$

We introduce the parameter $\alpha=\min \left(\beta, \sigma_{1}, \sigma_{2}\right)$; estimate the right-hand of (4.13) using Cauchy's inequality and apply Theorem (4.1) to find:

$$
\left(\int_{\Omega_{1}^{k} \cup \Omega_{2}^{k}}\left|\nabla u^{k}\right| d x+\beta \int_{\Gamma^{k}}\left(\left[u^{k}\right]\right)^{2} d s\right)^{1 / 2} \leq \alpha^{-1} \sqrt{C}\|f\|_{L^{2}(\Omega)} .
$$

It is evident that (4.9) - (4.11) follow immediately. The estimate (4.12) is obtained noting that $\max \left(\sigma_{1}, \sigma_{2}\right) \geq \sigma(x)>0$, and applying (4.10).

Remark 4.3. Noting that meas $\left(\Gamma^{k}\right)=0$ it follows immediately that $\left\{\nabla u^{k}\right\}$ and $\left\{\sigma^{k} \nabla u^{k}\right\}$ are elements of $L^{2}(\Omega)^{3}$. From Theorem 4.2 it follows that $\left\{\nabla u^{k}\right\}$ and $\left\{\sigma^{k} \nabla u^{k}\right\}$ are uniformly bounded sequences in $L^{2}(\Omega)^{3}$.

Remark 4.4. It is easily seen from Theorem 4.2 that $u^{k}$ lies in the space $S B V(\Omega)$. The distributional gradient of $u^{k}$ in $B V(\Omega)$ is a Radon measure denoted by $D u^{k}$. The absolutely continuous part is precisely $\nabla u^{k}$ and the singular part is confined to the two-phase interface and given by $-\left[u^{k}\right] n d H_{2} \mid \Gamma^{k}$. Summarizing, the distributional gradient has the representation

$$
D u^{k}=\nabla u^{k} d x-\left[u^{k}\right] n d H_{2}\left\lfloor\Gamma^{k} .\right.
$$

Here $d H_{2}\left\lfloor\Gamma^{k}\right.$ is 2 dimensional surface measure on $\Gamma^{k}$. The space $S B V$ has been applied to problems in continuum mechanics involving interfaces by several authors (see for example $[3],[11])$.

We now find the asymptotic equilibrium law for the heat flux. From Remark 4.3 we can find a subsequence $\left\{\sigma^{k^{\prime}} \nabla u^{k^{\prime}}\right\}$ of heat flux vectors converging weakly in $L^{2}(\Omega)^{3}$ to the vector field $M^{0}$ in $L^{2}(\Omega)^{3}$.

We observe that the space $H_{0}^{1}(\Omega)$ lies in the intersection of all the spaces $U^{k}$. Choosing $\psi$ in $H_{0}^{1}(\Omega)$ we have $[\psi]=0$ on phase interfaces and for every element in $\left\{\sigma^{k^{\prime}} \nabla u^{k^{\prime}}\right\}_{k^{\prime}}$ we have:

$$
\int_{\Omega} \sigma^{k^{\prime}} \nabla u^{k^{\prime}} \cdot \nabla \psi d x=\int_{\Omega} \psi f d x .
$$

Passing to the limit we find

$$
\int_{\Omega} M^{\circ} \cdot \nabla \psi d x=\int_{\Omega} \psi f d x, \text { for any } \psi \text { in } H_{0}^{1}(\Omega) .
$$

Equation (4.17) gives the homogenized equilibrium law for the heat flux. To finish the proofs of Theorems 2.1 and 2.3 we identify $M^{\circ}$; this is the goal of Sections 5 and 6 . 


\section{Characterization of the Two Scale Limit of the Tempera- tures}

The estimates given in the previous Section together with the two scale convergence introduced by Nguetseng [17] are used to characterize the two scale limit of the sequence of temperature fields $\left\{u^{k}\right\}$.

We introduce the space $L_{p}^{2}$ of $Q$-periodic functions in $L_{1 \text { oc }}^{2}\left(\mathbb{R}^{3}\right)$.

Definition 5.1. A sequence of functions $\left\{v^{k}\right\}$ in $L^{2}\left(\Omega ; L_{p}^{2}\right)$ two scale converges to the limit $v^{\circ}$ in $L^{2}\left(\Omega ; L_{p}^{2}\right)$ if

$$
\int_{\Omega} v^{k}(x) w(k x) \delta(x) d x \rightarrow \int_{\Omega \times Q} v^{\circ}(x, y) w(y) \delta(x) d x d y
$$

for all $w$ in $L_{p}^{2}$ and $\delta$ in $C_{c}^{\infty}(\Omega)$.

We observe from the definition that two scale convergence implies weak $L^{2}(\Omega)$ convergence of the sequence $\left\{v^{k}\right\}$ to the function $\int_{Q} v^{\circ}(x, y) d y$.

From Theorem 4.2 the sequence $\left\{u^{k}\right\}$ is uniformly bounded in $L^{2}(\Omega)$. It follows from the two scale convergence Theorem of Nguetseng [17] that there exists a two scale convergent subsequence (also denoted by $\left.\left\{u^{k}\right\}\right)$ converging to $u^{0}(x, y)$ in $L^{2}\left(\Omega ; L_{p}^{2}\right)$.

The limit is characterized in the following

Theorem 5.2. The two scale limit is a function of the macroscopic variable only, i.e.

$$
u^{\circ}(x, y)=u^{\circ}(x)
$$

Proof: For any $Q$-periodic vector field $\psi$ in $H^{1}(Q)^{3}$ and for any function $\delta$ in $C_{c}^{\infty}(\Omega)$ one has the identity:

$$
\begin{aligned}
& \int_{\Omega} u^{k}(\operatorname{div} \psi(k x)) \delta(x) d x=\int_{\Gamma^{k}}\left[u^{k}\right] n \cdot \psi(k x) \delta(x) d s \\
& -\int_{\Omega} u^{k} \psi(k x) \cdot \nabla \delta d x-\int_{\Omega} \delta(x) \psi(k x) \cdot \nabla u^{k} d x .
\end{aligned}
$$

Setting $y=k x$ we have $\operatorname{div} \psi(k x)=k \operatorname{div}_{y} \psi(k y)$ where $\operatorname{div}_{y}$ is the divergence with respect to the $y$-variable. Substitution into $(5.3)$ gives

$$
\begin{aligned}
& \int_{\Omega} u^{k}\left(\operatorname{div}_{y} \psi(k x)\right) \delta d x \\
& =k^{-1}\left\{\int_{\Gamma^{k}}\left[u^{k}\right] n \cdot \psi(k x) \delta(x) d s-\int_{\Omega} u^{k} \psi(k x) \cdot \nabla \delta d x-\int_{\Omega} \delta(x) \psi(k x) \cdot \nabla u^{k} d x\right\} .
\end{aligned}
$$


We apply Cauchy's inequality to the first term in (5.4) together with the estimate (4.11) and rescaling to obtain

$$
\left|k^{-1} \int_{\Gamma^{k}}\left[u^{k}\right] n \cdot \psi(k x) \delta(x) d s\right| \leq k^{-1 / 2} C\|\delta\|_{L^{\infty}(\Omega)}\left(\int_{\gamma^{1}}|\psi|^{2} d s\right)^{1 / 2} .
$$

From estimates (4.9) and (4.10) it follows that the last two terms in (5.4) are of order $k^{-1}$. Passing to a subsequence if necessary in (5.4) and applying the two-scale limit theorem of Nguetseng [17] gives:

$$
\int_{\Omega \times Q} u^{0}(x, y) \operatorname{div}_{y} \psi(y) \delta(x) d x d y=0,
$$

for any periodic $\psi$ function in $H^{1}(Q)$, and the theorem is proved. It follows form the remark after Definition 5.1 that the subsequence $\left\{u^{k}\right\}$ converges weakly in $L^{2}(\Omega)$ to the temperature $u^{\circ}(x)$.

\section{The Constitutive Relation for Heat Conduction in Fine Phase Mixtures}

In this Section we find the linear constitutive law relating the weak limit of the heat flux vectors $M^{\circ}$ to the weak limit of the temperatures $u^{\circ}(x)$. The constitutive law describes heat transport in the fine phase mixture. Under suitable hypothesis on the geometry of the mixture, we show that the weak limit of the temperatures $u^{0}(x)$ lies in the space $H^{1}(\Omega)$. To this end we introduce:

Theorem 6.1. For all test fields $\delta$ in the space $C_{c}^{1}(\Omega)$ and for all vectors $\xi$ in $\mathbb{R}^{3}$ we have:

$$
\int_{\Omega} u^{0} \mathbf{c} \xi \cdot \nabla \delta d x=-\int_{\Omega} \delta M^{0} \cdot \xi d x
$$

Here $\mathbf{c}$ is the constant tensor introduced in Section 1 given by

$$
\mathbf{c}=\sigma_{1} \mathbf{g}^{1}+\sigma_{2} \mathbf{g}^{2}
$$

It is evident from the definition of the tensors $\mathbf{g}^{1}$ and $\mathbf{g}^{2}$ (see equations (2.7) and (2.8)) that the tensor $\mathbf{c}$ is positive semidefinite.

Remark 6.2. Theorem 6.1 delivers the homogenized constitutive law:

$$
M^{\circ}=\mathbf{c} \nabla u^{0}
$$

It is evident from Theorem 6.1 that heat is transported only along directions perpendicular to the kernel of $\mathbf{c}$ and that $u^{0}$ has $L^{2}$ directional derivitaves for all such directions. 
The definiteness of $\mathbf{c}$ depends upon the geometry of the composite as addressed in Section 7 . When $\mathbf{c}$ is positive definite we have:

Corollary 6.3. The limit $u^{\circ}$ lies in $H^{1}(\Omega)$ and the distributional gradient of $u^{0}(x)$ is given by

$$
\partial_{x_{i}} u^{0}=M^{0} \cdot \mathbf{c}^{-1} e^{i}, \quad i=1,2,3
$$

where $e^{i}, i=1,2,3$ denotes an orthonormal system in $\mathbb{R}^{3}$. Moreover the distributional gradients for the sequence $\left\{u^{k}\right\}$ converge weakly in the sense of Radon measures, to $\nabla u^{\circ}$, i.e.,

$$
D u^{k}-\nabla u^{0} d x
$$

Proof of Theorem 6.1: The structure of this proof follows that of Tartar [19] for perfectly bonded two-phase conductors. Extending the corrector $\phi^{k}$ defined in Section 2 by periodicity to $\mathbb{R}^{3}$ we introduce a test $\delta$ in $C_{c}^{1}(\Omega)$ and set $\psi=\delta \phi^{k}$ in the variational formulation (1.8) to obtain

$$
\int_{\Omega_{1}^{k} \cup \Omega_{2}^{k}} \sigma^{k}(x) \nabla u^{k} \cdot \nabla\left(\delta \phi^{k}\right)+\beta \int_{\Gamma^{k}}\left[u^{k}\right]\left[\phi^{k}\right] \delta d s=\int_{\Omega} \delta \phi^{k} f d x
$$

Noting that the equation $\operatorname{div}\left(\sigma^{k} \nabla \phi^{k}\right)=0$ holds in the sense of regular distributions in each phase we multiply by $\delta u^{k}$ and integrate by parts using appropriate Green's Theorem [20] to obtain

$$
-\int_{\Omega_{1}^{k} \cup \Omega_{2}^{K}} \nabla\left(\delta u^{k}\right) \cdot \sigma^{k} \nabla \phi^{k} d x+\int_{\Gamma^{k}}\left[u^{k}\right] n \cdot \sigma^{k} \nabla \phi^{k} \delta d s=0 .
$$

In view of the jump condition (3.24) we write $(6.7)$ as

$$
\int_{\Omega_{1}^{K} \cup \Omega_{2}^{k}}\left(u^{k} \nabla \delta \cdot \sigma^{k} \nabla \phi^{k}+\delta \nabla u^{k} \cdot \sigma^{k} \nabla \phi^{k}\right) d x+\beta \int_{\Gamma^{k}}\left[u^{k}\right]\left[\phi^{k}\right] \delta d s=0 .
$$

Subtracting (6.8) from (6.6) gives

$$
\int_{\Omega_{1}^{k} \cup \Omega_{2}^{k}} \phi^{k} \sigma^{k}(x) \nabla u^{k} \cdot \nabla \delta d x-\int_{\Omega_{1}^{k} \cup \Omega_{2}^{k}} u^{k} \nabla \delta \cdot \sigma^{k} \nabla \phi^{k} d x=\int_{\Omega} \delta \phi^{k} f d x .
$$

Noting that meas $\left(\Gamma^{k}\right)=0$ and in view of Remark 4.3 we may write $(6.9)$ as

$$
\int_{\Omega} \phi^{k} \sigma^{k}(x) \nabla u^{k} \cdot \nabla \delta d x-\int_{\Omega} u^{k} \nabla \delta \cdot \sigma^{k}(x) \nabla \phi^{k} d x=\int_{\Omega} \delta \phi^{k} f d x .
$$

We now identify the limit of each term in (6.10) as $k$ goes to $\infty$ Recalling that $\phi^{k}=$ $k^{-1} \psi^{k}(k x)+\xi \cdot x$, elementary estimates show that there exists a constant $C_{0}$ such that

$$
\left\|\phi^{k}-\xi \cdot x\right\|_{L^{2}(\Omega)}=\left\|k^{-1} \psi^{k}(k x)\right\|_{L^{2}(\Omega)} \leq C_{0}\left\|k^{-1} \psi^{k}(y)\right\|_{L^{2}(Q)}
$$


It is evident from (6.11) and Theorem 3.7 that $\phi^{k} \rightarrow \xi \cdot x$ strongly in $L^{2}(\Omega)$. Recalling that the sequence $\sigma^{k} \nabla u^{k}$ converges weakly in $L^{2}(\Omega)^{3}$ to $M^{0}$ we find that the first term satisfies

$$
\lim _{k \rightarrow \infty} \int_{\Omega} \sigma^{k}(x) \nabla u^{k} \cdot \nabla \delta \phi^{k} d x=\int_{\Omega}\left(M^{\circ} \cdot \nabla \delta\right) \xi \cdot x d x
$$

Passing to the limit in the last term gives

$$
\lim _{k \rightarrow \infty} \int_{\Omega} \delta \phi^{k} f d x=\int_{\Omega} \delta \xi \cdot x f d x
$$

To pass to the limit in the middle term of $(6.10)$ we enlist the aid of a simple modification of Theorem 2 introduced in Nguetseng [17].

Proposition 6.5. Given the sequences $\left\{g^{k}\right\},\left\{w_{k}\right\}$ such that $\left\|g^{k}\right\| \leq C, g^{k}$ two scale converges to $g^{0}(x, y)$ and $w_{k} \rightarrow w_{\infty}$ strongly in $L_{p}^{2}(Q)$ then:

$$
\int_{\Omega} g^{k} v(x) w_{k}(k x) d x \rightarrow \int_{\Omega \times Q} g^{0}(x, y) v(x) w_{\infty}(y) d x d y
$$

for all functions $v$ in $C_{c}^{1}(\Omega)$.

Proof: As $k$ tends to infinity elementary estimates show there exists a constant $C_{0}$ independent of $k$ for which

$$
\left\|w_{k}(k x)-w_{\infty}(k x)\right\|_{L^{2}(\Omega)} \leq C_{0}\left\|w_{k}(y)-w_{\infty}(y)\right\|_{L^{2}(Q)} .
$$

From the hypothesis on $\left\{w_{k}\right\}$ and (6.15) is evident that

$$
\lim _{k \rightarrow \infty}\left\|w_{k}(k x)-w_{\infty}(k x)\right\|_{L^{2}(\Omega)}=0 .
$$

We write

$$
\begin{aligned}
& \left|\int_{\Omega} g^{k} v w_{k}(k x) d x-\int_{\Omega x Q} g^{\circ}(x, y) v w_{\infty}(y) d x d y\right| \leq \\
& \left|\int_{\Omega} g^{k} v\left(w_{k}(k x)-w_{\infty}(k x)\right) d x\right|+\left|\int_{\Omega} g^{k} v w_{\infty}(k x) d x-\int_{\Omega x Q} g^{\circ}(x, y) v w_{\infty}(y) d x d y\right| .
\end{aligned}
$$

The first term on the right hand side of (6.17) vanishes due to (6.16) and the uniform $L^{2}$ bound on $\left\{g^{k}\right\}$. The second term vanishes as the sequence $g^{k}$ two scale converges to $g^{\circ}(x, y)$ and the Proposition is established.

Passing to a subsequence if necessary we have from Theorem 5.2 that the two-scale limit of $u^{k}$ is $u^{\circ}(x)$. On the other hand $\sigma^{k}(x) \nabla \phi^{k}=\sigma(k x)\left(\nabla_{y} \psi^{k}(k x)+\xi\right)$ and from Lemma (3.2) it follows that

$$
\sigma(y)\left(\nabla_{y} \psi^{k}(y)+\xi\right) \rightarrow \sigma(y)\left(\nabla_{y} \psi^{\infty}+\xi\right)
$$


strongly in $L^{2}(Q)^{3}$. It is evident that the sequences $\left\{u^{k}\right\}$ and $\left\{\sigma^{k}(x) \nabla \phi^{k}\right\}$ satisfy the hypothesis of Proposition 6.5. Thus applying the Proposition to the middle term we find

$$
\begin{aligned}
& \lim _{k \rightarrow \infty} \int_{\Omega} u^{k} \nabla \delta \cdot \sigma^{k}(x) \nabla \phi^{k} d x= \\
& \quad=\int_{\Omega x Q} u^{0}(x) \nabla \delta \cdot \sigma(y)\left(\nabla_{y} \psi^{\infty}+\xi\right) d x d y \\
& \quad=\int_{\Omega} u^{\circ}(x) \mathbf{c} \xi \cdot \nabla \delta d x .
\end{aligned}
$$

Here we have used that $\mathbf{c}$ defined by (2.8) admits the representation

$$
\mathbf{c} \xi=\sigma_{1} \int_{Q_{1}^{1}}\left(\nabla_{y} \psi_{1}^{\infty}+\xi\right) d y+\sigma_{2} \int_{Q_{2}^{1}}\left(\nabla_{y} \psi_{2}^{\infty}+\xi\right) d y .
$$

Thus we obtain

$$
\int_{\Omega}\left(M^{\circ} \cdot \nabla \delta\right) \xi \cdot x d x-\int_{\Omega} u^{\circ}(x) \mathbf{c} \xi \cdot \nabla \delta d x=\int_{\Omega} \delta \xi \cdot x f d x
$$

and the theorem follows in view of (4.17).

Equation (6.4) of Corollary 6.3 follows from Theorem 6.1 by choosing $\xi=\mathbf{c}^{-1} e^{i}$. To establish the convergence we note that $u^{k}$ lies in $B V(\Omega)$ and for any $\psi$ in $C_{c}^{1}(\Omega)$

$$
\int_{\Omega} u^{k} \operatorname{div} \psi d x=-\int_{\Omega} \psi \cdot d\left[D u^{k}\right] .
$$

The Corollary follows from the weak convergence of $u^{k}$ to $u^{\circ}$.

\section{Stationary Thermal Transport Equation for Fine Phase Mixtures}

The homogenized equation for heat transport follows immediately from the results of the last Section and from (4.17). Indeed, $u^{\circ}$ is seen to be a solution of the homogenized problem:

$$
\int_{\Omega} \mathbf{c} \nabla u^{0} \cdot \nabla \psi d x=\int_{\Omega} \psi f d x
$$

for all $\psi$ in $H_{0}^{1}(\Omega)$, and Theorem 2.1 follows.

The positivity of the tensor $\mathbf{c}$ is governed by the geometry of the two phase interface. As an example we consider an isotropic suspension of particles. We assume that $Q_{1}^{1}$ is the connected matrix, that $Q_{2}^{1}$ is the particle phase and that the particles are isolated. We 
observe that this geometry satisfies the hypotheses (H1) and (H2) and note as in Remark 3.7 that $\mathbf{g}^{2}=0$. Thus,

$$
\mathbf{c}=\sigma_{1} \mathbf{g}^{1}
$$

For isotropic suspensions particles for which there exists a positive lower bound on the inter-particle distance, the estimates of Bruno [8] and Torquato and Rubinstein [21] show that $\mathbf{g}^{1}$ is positive and the positivity of $\mathbf{c}$ follows

As a second example we consider a laminar geometry with layer normal given by $e_{1}$ and thickness of phase one and two given by $\theta_{1}$ and $\theta_{2}$ respectively $\left(\theta_{1}+\theta_{2}=1\right)$. Calculation shows that the matrices $\mathbf{g}^{1}$ and $\mathbf{g}^{2}$ are diagonal and given by

$$
\mathbf{g}^{1}=\left(\begin{array}{ccc}
0 & & \\
& \theta_{1} & \\
& & \theta_{1}
\end{array}\right), \quad \mathbf{g}^{2}=\left(\begin{array}{ccc}
0 & & \\
& \theta_{2} & \\
& & \theta_{2}
\end{array}\right)
$$

and the resulting tensor $\mathbf{c}=\sigma_{1} \mathbf{g}^{1}+\sigma_{2} \mathbf{g}^{2}$ is not positive definite. The local conductivity of the 1 periodic laminate is written $\sigma(x)$. Thus if one considers a sequence of successively finer laminates $\sigma(k x)$ with associated temperature fields $u^{k}$, it is no longer evident that the limit temperature field $u^{\circ}$ belongs to $H^{1}(\Omega)$. However from Theorem 6.1 it does follow that the distributions $\partial_{x_{2}} u^{\circ}$ and $\partial_{x_{2}} u^{\circ}$ are elements of $L^{2}(\Omega)$.

More generally, the recent result of Briane [7] can be applied to establish positivity of the tensor $\mathbf{c}$ for any composite satisfying (H1) and (H2). Application of Proposition 5.1 and Remark 5.2 in [7] to the tensor $\mathbf{g}^{1}$ shows that it is positive and the positivity of $\mathbf{c}$ follows.

\section{Strong Convergence of Temperatures for Connected Phase Geometries}

In this section we suppose material one is connected according to the hypotheses (H1) and (H2). We observe that the region occupied by material two (i.e., $Q_{2}^{1}$ ) may have a finite number of connected and disconnected components.

We state the following:

Theorem 8.1. There exists a limit $u^{\circ}$ in $H_{0}^{1}(\Omega)$ and a subsequence $\left\{u^{k^{\prime}}\right\}$ such that $u^{k^{\prime}} \rightarrow u^{\circ}$ strongly in $L^{2}(\Omega)$.

Proof: We recall that for each $k=1,2, \ldots$ the temperature $u^{k}$ lies in $U^{k}$ given by (1.7), i.e., $u^{k}=\left(u_{1}^{k}, u_{2}^{k}\right), u_{1}^{k}$ in $H^{1}\left(\Omega_{1}^{k}\right), u_{2}^{k}$ in $H^{1}\left(\Omega_{2}^{k}\right)$ with $u_{1}^{k}=0$ on $\partial \Omega_{1}^{k} \cap \partial \Omega$ and $u_{2}^{k}=0$ on $\partial \Omega_{2}^{k} \cap \partial \Omega$. Moreover we have from (4.9) and (4.10) that $\left\|u_{1}^{k}\right\|_{H^{1}\left(\Omega_{1}^{k}\right)} \leq C$ where the constant $C$ does not depend upon $k$. We introduce $\tilde{u}_{1}^{k}$ defined by $\tilde{u}_{1}^{k}=\left(u_{1}^{k}, 0\right)$.

Noting that we have satisfied the hypotheses of the Compactness Lemma of Allaire, Murat, and Nandakumar, (see Lemma A.3 of [1]) we apply it to find a subsequence $\tilde{u}_{1}^{k^{\prime}}$ for 
which there exists a function $u^{0}$ in $H_{0}^{1}(\Omega)$ such that:

$$
\tilde{u}_{1}^{k^{\prime}} \rightarrow \theta_{1} u^{\circ} \quad \text { weakly in } L^{2}(\Omega)
$$

Here $\theta_{1}$ is the volume fraction of conductor one in the composite. Moreover, Lemma A.3 of [1] asserts that for any sequence $v^{k^{\prime}}$ in $L^{2}\left(\Omega_{1}^{k^{\prime}}\right)$ for which $\tilde{v}^{k^{\prime}}=\left(v_{1}^{k^{\prime}}, 0\right) \rightarrow \theta_{1} v$ weakly in $L^{2}(\Omega)$, that:

$$
\int_{\Omega_{1}^{k^{\prime}}} u_{1}^{k^{\prime}} v_{1}^{k^{\prime}} d x \rightarrow \int_{\Omega} \theta_{1} u^{0} v d x
$$

From $(8,1)$ and $(8,2)$ it is evident that

$$
\left\|u_{1}^{k^{\prime}}-u^{0}\right\|_{L^{2}\left(\Omega_{1}^{k}\right)} \rightarrow 0 .
$$

Next, we appeal to Lemma 6 of Lene and Leguillon [15] which states that for all $\psi=\left(\psi_{1}, \psi_{2}\right)$ in $U^{k}$ there exists a constant $C$ independent of $k$, such that

$$
\left\|\psi_{2}\right\|_{L^{2}\left(\Omega_{2}^{k}\right)}^{2} \leq C\left(\left\|\psi_{1}\right\|_{L^{2}\left(\Omega_{1}^{k}\right)}^{2}+k^{-1} \int_{\Gamma^{k}}([\psi])^{2} d s+k^{-2} \int_{\Omega_{1}^{k} \cap \Omega_{2}^{k}}|\nabla \psi|^{2} d x\right) .
$$

Noting that $\psi=\left(u_{1}^{k^{\prime}}-u^{\circ}, u_{2}^{k^{\prime}}-u^{\circ}\right)$ lies in $U^{k^{\prime}}$ and application of (8.4) gives

$$
\begin{gathered}
\left\|u_{2}^{k^{\prime}}-u^{0}\right\|_{L^{2}\left(\Omega_{2}^{k^{\prime}}\right)}^{2} \leq C\left(\left\|u_{1}^{k^{\prime}}-u^{0}\right\|_{L^{2}\left(\Omega_{1}^{k^{\prime}}\right)}^{2}+k^{-1} \int_{\Gamma^{k}}\left(\left[u^{k^{\prime}}-u^{0}\right]\right)^{2} d s+\right. \\
\left.k^{-2} \int_{\Omega_{1}^{k} \cap \Omega_{2}^{k}}\left|\nabla u^{k^{\prime}}-\nabla u^{0}\right|^{2} d x\right)
\end{gathered}
$$

From (8.3) and the estimates (4.10) and (4.11), it follows that the right-hand side of (8.5) vanishes in the limit as $k^{\prime}$ tends to infinity, hence,

$$
\left\|u_{2}^{k^{\prime}}-u^{0}\right\|_{L^{2}\left(\Omega_{2}^{k^{\prime}}\right)} \rightarrow 0
$$

From (8.3) and (8.6) it follows that $u^{k^{\prime}}$ converges strongly in $L^{2}(\Omega)$ to $u^{\circ}$.

We remark that arguments identical to those given in Sections 6 and 7 show that the function $u^{\circ}$ solves the local equation (2.14). Uniqueness of the solution follows from the strict ellipticity of the operator $\nabla \cdot \mathbf{c} \nabla$. It is evident that the whole sequence $\left\{u^{k}\right\}$ converges to $u^{\circ}$ in $L^{2}(\Omega)$ and Theorem 2.3 is proved.

\section{Acknowledgments}

This work was supported by NSF grant DMS-9403866. 


\section{References}

[1] G. Allaire and F. Murat, Homogenization of the Neumann problem with nonisolated holes, Asymptotic Analysis 7 (1993), pp. 81-95.

[2] L. Ambrosio and E. DeGeorgi, Un nuovo tipo di funzionale del calcolo delle variazioni, Atti Accad. Naz. Lincei Rend. Cl. Sci. Fix. Mat. Natur. 82 (1988), pp. 199-210.

[3] H. Barroso, G. Bouchitte, G. Buttazzo, and I. Fonseca, Relaxation of bulk and interfacial energies, Preprint, Department of Mathematics, Carnegie Mellon University, Pittsburgh (1994).

[4] A. Bensoussan, J.L. Lions, and G. Papanicolaou, Asymptotic Analysis for Periodic Structures, (Amsterdam: North-Holland 1979).

[5] Y. Benveniste, Effective thermal conductivity of composites with a thermal contact resistance between the constituents: Nondulute case, J. Appl. Phys. 61 (1986), pp. $2840-2844$.

[6] H. Bhatt, K.Y. Donaldson, and D.P.H. Hasselman, Role of the interfacial thermal barrier in the effective thermal diffusivity/conductivity of SiC-Fiber-reinforced reaction-bonded silicon nitride, J. Am. Ceram. Soc. 73 (1990), pp. 312-316.

[7] M. Briane, The Poincaré-Wirtinger's inequality for the homogenization in perforated domains, Preprint.

[8] F.A.L. Dullien, Porous Media Fluid Transport and Pore Structure, Academic Press, New York (1979).

[9] O.P. Bruno, The effective conductivity of strongly heterogeneous composites, Comm. Pure Appl. Math. 43 (1990), pp. 769-207.

[10] L.C. Evans and R. Gariepy, measure Theory and Fine Properties of Functions, CRC Press, Boca Raton (1992).

[11] I. Fonseca and G. Francfort, Relaxation in $B V$ versus quasi-convexification in $W^{1, p}$ : a model for the interaction between damage and fracture. Preprint, Department of Mathematics, Carnegie Mellon University, Pittsburgh (1994).

[12] A.G. Every, Y. Tzou, D.H.P. Hasselman and R.RaJ, The effect of particle size on the thermal conductivity of $Z n S /$ diamond composites, Acta Metal. Mater 40 (1992), pp. 123-129.

[13] K. Golden and G. Papanicolaou, Bounds for effective parameters of heterogeneous media by analytic continuation, Commun. Math. Phys. 90 (1983), pp. 473-491. 
[14] F. Lene And D. Leguillon, Homogenized constitutive law for a partially cohesive composite material, Int. J. Solids Structures, 18 (1982), pp. 443-458.

[15] F. Lene and D. Leguillon, Étude de l'influence d'un glissement entre les constituants d'un matériau composite sur ses coefficients de comportement effectifs, Journal de Mécanique, 20 (1981), pp. 509-536.

[16] R. Lipton and B. Vernescu, Composites with imperfect interface, IMA Preprint, (1995).

[17] G. Nguetseng, A general convergence result for a functional related to the theory of homogenization, SIAM J. Math. Anal. 20 (1989), pp. 608-623.

[18] E. Sanchez Palencia, Non-Homogeneous Media and Vibration Theory, SpringerVerlag, Berlin-Heidelberg-New York, 1980.

[19] L. Tartar, Cours Peccot au Collège de France, Unpublished (March 1977).

[20] R. Temam, Navier-Stokes Equations Amsterdam, North Holland, (1984).

[21] S. Torquato and J. Rubinstein, Improved bounds on the effective conductivity of high-contrast suspensions, J. Appl. Phys. 69 (1991), pp. 7118-7125. 